\title{
Restoration Experience Measurement Methods in Contact with Green Open Spaces
}

\author{
Fahimeh Malekinezhad and Hasanuddin bin Lamit \\ Faculty of Built Environment, Universiti Teknologi Malaysia, \\ Skudai, Johor, Malaysia
}

\begin{abstract}
Contact with nature is suggested to be as an important resource for human health and well-being. Stress alleviation and reduction of mental fatigue are main instances of improvement of human health and well-being in contact with nature. Although the term restoration experience in contact with nature has been used repeatedly for concepts of stress alleviation and reduction of mental fatigue, these refer to different sets of theories and research studies. In this paper, by reviewing the nature-health related theories of Biophilia, Stress Recovery Theory, Attention Restoration Theory and Supportive Environment Theory (SET), the similarities and differences between these two concepts have been discussed. Moreover, the measurement approach instruments based on different study methodologies and aims are provided. This paper could provide information for future studies with focus on factors to be of benefit for restoration experience in design and planning of natural landscape environments with the aim of health and well-being development.
\end{abstract}

Keywords: restoration experience; Biophilia Hypothesis; Stress Recovery Theory (SRT); Attention Restoration Theory (ART); Supportive Environment Theory (SET)

\section{1-Introduction}

Passive and active interactions with nature positively contribute to human health and well-being outcomes. The beneficial impact of contact with nature is on human physical, social and mental health and well-being (Abraham et al., 2010). The indirect effect of nature is by improvement of socialization and physical activity levels (Francis et al., 2012; De Vries et al., 2013). Direct effect of nature on human mental health is through restoration experience by exposure to natural elements of vegetation and water. This study focuses on the aspects that can be associated with restoration experience via passive and active interactions with nature and its components.

Restoration experience is the renewal of human capacities through exposure to nature. In environmental health related studies, restoration can be proceed through recovery of cognitive fatigued and stress alleviation. It is the process by which one "moving from a depleted state to a restored state" Johnsen (2013). In meeting with everyday life demands, people's physiological, psychological and emotional resources become declined (Ulrich et al., 1991; Kaplan and Kaplan, 1989; Hartig, 2007; Hartig and Staats, 2005; Honold et al., 2015). Therefore, people need to rejuvenate these resources regularly in natural environments. Otherwise, over time, lack of restoration can be lead to human health problems and threat human well-being (Hartig, 2007).

In literature, the term of restoration experience has been applied with interchangeable concepts such as stress recovery, cognitive functioning or mental fatigue restoration (Ulrich et al., 1991; Korpela et al., 2008; Kaplan and Kaplan, 1989). Restoration experience is the result of changes in human system from tension towards positive states. Nature-health related studies measured restoration experience through actual 
changes in human psycho-physiological and emotional state or through subjective self-reported surveys. Experience of restoration through contact with nature is well supported in nature related theories; however, there is a gap for classification of approaches in assessment of restoration and influential characteristics. Therefore, in this study, the related theories to beneficial effect of contact with nature on restoration experience and approaches in assessment of restoration and potential characteristics are discussed. In this paper, firstly, related theories to restoration in natural environments, restorative outcome measurement approaches and influential characteristics are discussed. Then, actual and subjective restorative outcomes in exposure to nature are explained. After that, the effect of direct and indirect interactions with nature on experience of restoration is presented. The paper will be concluded with a tabular presentation of restoration experience measurement methods.

\section{2-Theories of Restoration Experience}

The possible explanation on the beneficial effect of nature on human health and wellbeing is grounded in Biophilia hypothesis. The two prominent theories of Stress Recovery Theory (SRT) and Attention Restoration Theory (ART) have been developed based on this idea. Furthermore, the Supportive Environment Theory (SET) is the developed framework in supporting the green space influential characteristics that maintain human health. All of these theories are discussed as following and in Table 1.

\section{1-Biophilia Hypothesis}

The first theory that is developed on beneficial effect of contact with nature is Biophilia hypothesis and introduced by Wilson (1984). Based on this theory, restoration experience in natural environment refers to human evolutionary affiliation with nature. The rapid affective response to nature and its components have been embedded in biology of human inclination to attach with nature (Nisbet et al., 2011). Based on this evolutionary connection to nature, human have innate connection to nature components such as lakes, hills, soil, beaches, forests, garden, bird's song and colorful flowers. Through Biophilia hypothesis, studies have investigated the positive health-related outcomes of natural environments through the extent of people connectedness to nature (e.g. Haluza et al., 2014; Capaldi et al., 2014). The connectedness to nature scale (CNS) is the instruments that have developed and validated in measurement of positive outcomes of human emotional connection to the natural world (Mayer and Frantz, 2004). Researchers based on this viewpoint support the idea that a sense of connection to nature is a significant predictor of human health and subjective well-being (Nisbet et al., 2011; Perrin and Benassi, 2009).

\section{2-Stress Recovery Theory (SRT)}

SRT suggests that natural landscapes are places for stress alleviation (Han, 2003; Ulrich, 1984). If a person is experiencing a high level of stress, nature exposure rapidly elicits positive affect and declines negative feelings and stressful thoughts (Hartig, 2007; Ulrich et al., 1991). Recovery from stress can be manifested through beneficial changes in emotional states and in activity of physiological dimensions of stress response (Bratman et al., 2015). Based on this theory, affective response in contact with natural environments can be evoked by certain environmental characteristics as mild complexity, focal point to attract attention and natural features such as exposure to vegetation and water (Hartig, 2007). 
Table 1 - Summary of Restoration Experience Measurement Approaches \& Theories

\begin{tabular}{|c|c|c|c|}
\hline $\begin{array}{l}\text { Measurement } \\
\text { Instrument }\end{array}$ & $\begin{array}{l}\text { Underpinni } \\
\text { ng Theory }\end{array}$ & Measurement Factors & Details \\
\hline $\mathrm{CNS}$ & $\begin{array}{l}\text { Biophilia } \\
\text { Hypothesis }\end{array}$ & Connection to nature & $\begin{array}{l}\text { General restoration and well- } \\
\text { being outcomes }\end{array}$ \\
\hline $\begin{array}{l}\text { Body } \\
\text { Examinations }\end{array}$ & SRT & Physiology system & Actual restoration \\
\hline POMS & SRT & Emotional state & Actual restoration \\
\hline PANAS & SRT & Emotional state & Actual restoration \\
\hline ZIPERS & SRT & Emotional state & Actual restoration \\
\hline ANT & ART & Cognitive functioning & Actual restoration \\
\hline RST & ART & Cognitive functioning & Actual restoration \\
\hline $\mathrm{DPT}$ & ART & Cognitive functioning & Actual restoration \\
\hline PRS-16 items & ART & Perceived restorativeness & Original version \\
\hline PRCS-15 items & ART & Perceived restorativeness & Applicable to use with children \\
\hline $\begin{array}{l}\text { PRCQ-24 } \\
\text { items }\end{array}$ & ART & Perceived restorativeness & $\begin{array}{l}\text { Restorativeness in zoos by } \\
\text { visitors }\end{array}$ \\
\hline $\begin{array}{l}\text { PDRQ-30 } \\
\text { items }\end{array}$ & ART & Perceived restorativeness & $\begin{array}{l}\text { Vacation experiences in the } \\
\text { tourism setting }\end{array}$ \\
\hline PRS-11 items & ART & Perceived restorativeness & $\begin{array}{l}\text { Shorter version of PRS in Italian } \\
\text { and English }\end{array}$ \\
\hline RCS-22 items & ART & Perceived restorativeness & $\begin{array}{l}\text { Restorative components of } \\
\text { environments }\end{array}$ \\
\hline PSD & SET & Green space characteristics & $\begin{array}{l}\text { Supportive qualities to maintain } \\
\text { restoration }\end{array}$ \\
\hline $\begin{array}{l}\text { SRPRS- } 12 \\
\text { items }\end{array}$ & ART & Cognitive restoration & Actual restoration \\
\hline RS- 17 items & $\begin{array}{l}\text { ART and } \\
\text { SRT }\end{array}$ & $\begin{array}{l}\text { Emotion, physiology, } \\
\text { cognition, and behavior }\end{array}$ & Actual restoration \\
\hline SRRS-8 items & $\begin{array}{l}\text { ART and } \\
\text { SRT }\end{array}$ & $\begin{array}{l}\text { Emotion, physiology, } \\
\text { cognition, and behavior }\end{array}$ & Actual restoration \\
\hline RSS- 9 items & ART & $\begin{array}{l}\text { Overall experience of } \\
\text { restoration }\end{array}$ & Actual restoration \\
\hline ROS-6 items & $\begin{array}{l}\text { SRT and } \\
\text { ART }\end{array}$ & $\begin{array}{l}\text { Relaxation and calmness, } \\
\text { attention restoration, clearing } \\
\text { thoughts }\end{array}$ & Subjective restoration \\
\hline POS-9 items & $\begin{array}{l}\text { SRT and } \\
\text { ART }\end{array}$ & $\begin{array}{l}\text { Relaxation and calmness, } \\
\text { attention restoration, clearing } \\
\text { thoughts, subjective vitality } \\
\text { and self-confidence }\end{array}$ & Subjective restoration \\
\hline
\end{tabular}

Research based on actual stress restoration monitored the effect of nature contact on changes in human physiology system by measurement of blood pressure, heart rate, cortisol concentration and muscle tension as the major components of stress response. For instance, in a field experimental study, Tyrv ainen et al. (2014) measured cortisol concentration through salivary cortisol as a physiological indicator of stress response. It showed that the salivary cortisol level was significantly reduced after visit to environments with natural elements. The impact of nature experience on immediate improvement of individuals' mood state and emotional arousal was measured through several emotional self-reported instruments including of Profile of Mood States 
(POMS) (Park et al., 2011), Positive and Negative Affect Scale (PANAS) (Marselle et al., 2014) and Zuckerman Inventory or Personal Reactions (ZIPERS) (Ulrich, 1979).

\section{3-Attention Restoration Theory (ART)}

The focus of ART is on cognitive restoration in contact with nature Kaplan and Kaplan (1989). ART divides human attention to two parts as direct attention and indirect attention. The direct attention is the voluntary form of attention, which is employment of mind effort to focus on daily tasks. Sustain use of direct attention causes exhaustion of directed attention and leads to Directed Attentional Fatigue (DAF) or what so-called as mental fatigue (Kaplan, 1993, 1995). DAF decreases effectiveness in functioning (e.g, less ability to work without error), causes a variety of negative emotions (e.g, bad humor, irritability, impatience), increases stress in lifestyle and eventually leads to serious health related problems (Kaplan et al., 1993; Han, 2003). In contrast with direct attention, the indirect attention is without capacity limitation (Hartig et al., 1997a). Restoration experience in ART obtains by effortless attention in natural environments, which gives attentional system a time for recovery.

There are substantial evidences on the restorative value of nature experience in improvement of the capacity of direct attention and cognitive ability. Studies measure the impact of nature experience on cognitive functioning through employing a verity of directed-attention ability tests including proofreading a text for spelling and punctuation errors, clinical assessment procedures to detect neuropsychological problems and backwards digit-span tasks and Attention Network Task (ANT) (Berman et al., 2008). In an experimental study, Pilotti et al. (2014) found that viewing natural environments' video at the end of a working day significantly enhanced sustained attention and long-term memory performance. In a controlled laboratory experiment, Raanaas et al. (2011) showed that viewing of nature in office settings can constitute restorative experience and make differences on measures of attention capacity functioning; Reading Span Task (RST) and a Dual Processing Task (DPT).

\subsection{1-Perceived Restorativeness}

Perceived restorativeness is the concept, which has been developed, based on ART. It suggests that restoration experience becomes manifested through perceiving environments rich in restorativeness qualities such as 'Being away', 'Fascination', 'Extent' and 'Compatibility' (Han, 2003; Kaplan, 2001a).

- Being away: It provides environmental opportunity to free one's mind from everyday life demanding tasks, obligations, routines, purposes and thoughts. Studies emerged two factors from this concept as physically 'Being away' and mentally 'Being away' (Laumann et al., 2001; Bagot et al., 2015). The physically 'Being away' refers to transformation and geographical distance from where one is performing life routines and obligations. The mentally 'Being away' refers to a sense of psychologically away from everyday responsibilities. It has been suggested that restoration of attentional fatigue is unlikely to occur only with physical movement from everyday environment and continuing everyday cares and concerns (Kaplan, 1995). The essential situation for restoration of directed attention is where one is able to be away from life stressors, undesirable distractions and reminders of obligations (Kaplan, 1995). One can find restoration of mental fatigue even in an old environment when experiences it in a new way (Kaplan, 1995). 
- Fascination: It refers to the opportunity the environment provides to restore mental fatigue through observing fascinating objects. There are two types of description for fascinating objects in an environment known as soft fascinating objects and hard fascinating objects. The soft 'Fascination' refers to experience of pleasing objects such as walking in a natural setting and see clouds, snow patterns, sunsets, movement of leaves in the wind and their flickering in sunsets (Kaplan, 1995). An example of hard 'Fascination' can be when one keeps a look out on environmental or social threats like venomous animals or crime, which negatively evoke ones' attention. The soft 'Fascination' is most conductive to mental restoration as effortlessly hold attention and allows mind the time for recovery (Kaplan, 1995).

- Extent: The next construct in Attention Restoration Theory (ART) is 'Extent'. The 'Extent' refers to an environment that is rich enough in content and structure (Kaplan, 2001b). It can occupy one's mind to interesting and enjoyable objects for a long period (Herzog et al., 2003; Kaplan, 1995). This concept also has been described as experiencing the combination of 'connectedness' and 'scope' in an environment (Kaplan and Kaplan, 1989). The component of 'connectedness' refers to coherence and relatedness between environmental elements to each other and if the elements constitute to a larger structure (Pals et al., 2009). The component of 'scope' refers to the scale and capacity of the environment that provide enough to see and experience (Kaplan, 2001b). Environments by having both 'coherence' and 'scope' can occupy mind for a long time and support extended exploration that allow directed attention to take a rest (Herzog et al., 2003; Kaplan, 2001a).

- Compatibility: It refers to the match between the characteristics of the environment and a person goals and inclinations (Kaplan, 1995). In a compatible environment, there is no struggle. Environment supports what a person likes to do, and a person comfortably does on what is appropriate to the environment demands (Kaplan and Peterson, 1993).

In conceptualization of psychological restoration and perceived restorativeness, it has been explained that both concepts emphasize "the recovering aspects of places, which allow people to distract, to relax, to free their minds and to distance themselves from ordinary aspects of life" Scopelliti and Vittoria Giuliani (2004, p.423). The difference is that psychological restoration is the state of reduced mental fatigue and perceived restorativeness is the capacity of setting where mental fatigue is reduced (Han, 2003). Therefore, research based on perceived restorativeness approach evaluates people perception of the potential restorative capacity of physical environment (Ivarsson and \& Hagerhall, 2008). In link between nature and psychological restoration outcomes, perceived restorativeness also played a key role in shaping of positive outcomes. It has been used as the underlying mechanism to explain how nature can induce emotional well-being outcomes (Marselle et al., 2015, 2016) and better quality of life (Hipp et al., 2016).

\subsection{2-Perceived Restorativeness Scale (PRS)}

Perceived Restorativeness Scale (PRS) has become a dominant instrument for assessment of perceived restorativeness. It is a self-response measurement instrument, with four factors structure of 'Being away', 'Fascination', 'Extent', and 'Compatibility'. It was structured and developed based on framework of ART, in a series of studies by Hartig et al. (1997b, 1991, 1996, 1997a). Several measurement 
instruments have been developed based on its original version to measure the restorative components of different settings with differences on its dimensional structures. For example, Bagot (2004) developed Perceived Restorative Components Scale (PRCS15 items) with 5 factors of 'Fascination', 'physically being away', 'Compatibility', 'Psychologically being away' and 'Extent', which is applicable to use with children. Pals et al. (2009) developed Perceived Restorative Characteristics Questionnaire (PRCQ), with 24-items and five factors of 'Fascination', 'Novelty', 'Escape', 'Coherence' and 'Compatibility' in measuring of the actual experiences of perceived restorative characteristics of zoos by visitors.

The Perceived Destination Restorative Quality (PDRQ) scale is another version, which developed by Lehto (2013) in examining measures of restorative properties of vacation experiences in the tourism setting. The proposed PDRQ includes 30-items and six dimensions of 'Extent', 'Fascination', 'Mentally away', 'Physically away', 'Discord' and 'Compatibility'. The PDRQ provided an instrument that facilitated to understand, which features and characteristics in a vocation destination contributed best to recovery from mental fatigue of vacationers. Pasini et al. (2014) developed a shorter version of PRS (in both Italian and English) with 11 items on factors of 'Being away', 'Fascination', 'Coherence' and 'Scope'.

The Restorative Components Scale (RCS22 items) is developed by Laumann et al. (2001) as a rating scale in measuring of restorative components of environments with five factors structure of 'Novelty', 'Escape', 'Extent', 'Fascination' and 'Compatibility'. In this scale, the component of 'Being away' is divided into 'Escape' and 'Novelty', which referred to the two aspects of 'Being away' quality as conceptually and physically distance from everyday life. This scale overcomes the concerns attributed to the original PRS, including the psychometric construction of items and factorial construct of the scale (e.g. Pasini et al., 2014; Hartig et al., 1997a). The item development in this scale does not emphasis on the aspects that make it specific for a particular environment.

Through literature review on psychological restoration in contact with nature, it has been found that studies assessed restoration experience through PRS scales, among stressed or mentally fatigued individuals (e.g. Tyrväinen et al., 2014). While, PRS scales are valid tools in evaluation of people's perception of restorativeness potential of settings and they are not suitable to monitor changes in actual restoration state (Marselle et al., 2015; Van den Berg et al., 2014).

\section{4-Supportive Environment Theory (SET)}

Development of SET is based on the restorative value of green spaces and factors that support psychological health (Grahn et al., 2010; Stoltz et al., 2016). Research based on SET suggested that in natural environments there are combinations of specific characteristics, which are supportive for psychological restoration (Grahn and Stigsdotter, 2010). These green space related characteristics has been so-called as Perceived Sensory Dimension (PSD) and are 'nature', 'culture', 'prospect', 'social', 'space', 'rich in species', 'refuge' and 'serene' Grahn and Stigsdotter (2010). These eight perceived sensory qualities was developed by several attempts on specification of green spaces characteristics between years of 1985 to 2010 (Grahn and Stigsdotter, 2010; Grahn et al., 2005; Grahn, 1991). The first and second generations of these 
characteristics were addressed in Grahn and his colleges studies (Grahn and Stigsdotter, 2010; Grahn et al., 2005; Grahn, 1991).

- Nature: The dimension of 'nature' has been described as an environment with some natural quality in the form of green surroundings like rows of trees, hedges, bushes, ponds, ditches, etc. This dimension consists of symbols of nature manifesting itself through its own condition such as free growing lawns, selfsown plants, mossgrown rocks, old paths. The experience includes a feeling of being in uncrowded, safe and relaxing environment. This dimension has been found in other related studies on open spaces (e.g. Herzele and Wiedemann, 2003). It has been labeled as 'wild' in the study by Grahn et al. (2005).

- Culture: This dimension also has been labeled as 'culture and history' or 'ornamental characteristic'. It is an environment containing traces of human culture. Environments with involving man-made or natural elements such as fountains, statues, exotic plants, ponds, ornamental plants, etc. It offers 'Fascination' with the passage of time through changing the texture and color of material (e.g. palnts) according to different weather conditions (Maikov et al., 2008). This dimension also has been found in other studies on green space qualities (Grahn et al., 2005; Herzele and Wiedemann, 2003).

- Social: The dimension of 'social' attributed to an environment where one can meet other people. The environment offers meeting place and equipped for social activities like places to sit, good lighting, sunny and shady places. It is a versatile space which are adaptable to many different functions or activities and offer gatherings in any way. It offers the experience that include feelings of good place in which people can amuse themselves, where people can meet and have pleasure. In other studies this dimension has been labeled as 'festive' (Grahn et al., 2005; Grahn, 1991), 'good services' (Kytta and Kahila, 2005) and 'facilities' (Herzele and Wiedemann, 2003).

- Space: The dimension of 'space or spacious' has been labeled as 'free', 'connectedness', 'boundless', 'extensive and 'coherent wholes'. It "must be perceived in a way that one may move freely, without being aware of the limited dimensions of the green space"(Herzele and Wiedemann, 2003, p.113). The area should offer a restful feeling of entering to another world and enable a person to stay for a longer time. This dimension in other related studies on green space qualities has been labeled as 'spaciousness' (Herzele and Wiedemann, 2003) and 'space and freedom' (Tyrväainen et al., 2007).

- Rich in Species: The dimension of 'rich in species' refers to wealth of plants and animals and diversity of nature. It described as an environment with a wide range of species of non-human life: variety of plants and animals (many birds, butterflies, flowers, etc) that draw attention. This dimension in other related studies to green space qualities labeled as 'lush' (Bjork et al., 2008; Grahn and Berggren-B̈̈rring, 1995), 'rich variety of species' and 'species richness' (De Jong et al., 2011; Grahn, 1991; Wolf et al., 2017).

- Refuge: The dimension of 'refuge' refers to an environment that is 'sanctuary', 'enclosed', 'secret' and 'secluded'. Areas enclosed by natural or man made landscape features like many bushes, higher vegetation, clumps of trees architectural elements to create different spaces and experiences. Environment provides opportunities of watching others and being active without being seen. Experiencing this dimension include a sense of comfort and privacy. In Appleton's refuge theory it is where people settled, seeking safe places where 
they could hide. This dimension in other related studies on green space qualities labeled as 'the pleasure garden' (Grahn et al., 2005).

- Prospect: The dimension of 'prospect' refers to an environment that is not enclosed with too many natural and man-made elements. 'Prospect' refers to the opportunity to see out with different types of views. In a green open space, it refereed to visual access to surrounding landscape and see what is going on around. Visibility is the main feature of this dimension that encourage a person to stay in an environment (Maikov et al., 2008). Having visual control over the surrounding is the aspect, which was highlighted in Appleton's Prospect theory (Grahn and Stigsdotter, 2010). This dimension in other related studies on green space qualities labeled as 'the common' (Grahn et al., 2005).

- Serene: The dimension of 'serene' refers to an environment that is 'silence' and 'calm'. It has natural sounds like the sound of wind, water, birds, insects and the like. It is an environment, which is clean with absent of weeds, disturbing people and traffic noise. The experience includes a feeling of being in a restful environment, which is safe and secure. This dimension in other related studies on green space qualities labeled as 'peaceful' (Grahn, 1991; Kytta and Kahila, 2005), 'quietness' (Herzele and Wiedemann, 2003) and 'peace and quiet' (Tyrväinen et al., 2007).

There are several studies with focus on green space qualities of PSD in association with perceived restorativeness characteristics and positive outcomes. Peschardt and Stigsdotter (2013) was the first study that showed PSD positively and strongly is related with perceived restorativeness characteristics in urban small parks. It showed that 'serene' and 'social' had the most associations in ratings of non-perceived stressed park users. In the forest stands, Stoltz et al. (2016) examined the stressreducing effect of these settings through examining the impact of five PSDs of 'serene', 'wild', 'species richness', 'space' and 'culture'. With exception of species richness dimension, restorative potential of forest settings was seen by the mediating effect of other qualities. In examining the health promoting effect of natural landscapes, Stigsdotter et al. (2017) explored the psychologically restorative effects of PSD qualities in a forest environment with designed rooms with eight PSD features. The most rated restorative settings were rooms with PSDs serene, nature, rich in species and refuge. In a visual assessment, Memari et al. (2017) showed that the PSD serene, nature and refuge were the most identified qualities for stress restoration.

Studies have used different objective and subjective measurement approaches, in highlighting the opportunities these green space related characteristics could provide for restoration experience. In a range of studies, assessment of green space characteristics has been carried out using the objective quantifiable measurement approaches. Through expert approach, a number of studies used Geographic Information System (GIS) to assess the presence/absence of each of these five characteristics within the participants' geocode residential addresses (e.g. Annerstedt et al., 2012; Van den Bosch et al., 2015; De Jong et al., 2012). Additionally to GISbased assessments, the area-aggregated self-report assessment method has been used in measuring of the green space characteristics within 510 min walking distance from the participant's residence area (De Jong et al., 2012, 2011). Although, these assessment approaches easily enabled monitoring of quantifiable nature characteristics and overcame single-source bias, the measurements only permit assessment of five characteristics out of eight qualities (Annerstedt et al., 2012; Van 
den Bosch et al., 2015). Moreover, studies have revealed that there are differences between perceived and objective approaches in landscape perception in related to people health conditions (e.g Francis et al., 2012). Mostly, they have advocated the importance of perceived environmental qualities rather than objective quantifiable measures for increasing better mental health outcomes (e.g. De Vries et al., 2013; Van Dillen et al., 2012; Francis et al., 2012; De Jong et al., 2011).

There are other researchers investigating the assessment of green space characteristics with eight PSDs. For instance, Peschardt and Stigsdotter (2013) asked two professional landscape architects to objectively evaluate the PSDs of nine small public urban green spaces (SPUGS) on-site. Qiu and Nielsen (2015) explained that experts assessment approach hold a dubious source to involve users' expression, ideas and feelings. Studies have shown the different viewpoints of experts and laypersons in perceiving of the landscape characteristics (Hofmann et al., 2012; Karmanov and Hamel, 2008). According to Grahn and Stigsdotter (2010), in a real-world experience, when seeking information on positive outcomes of nature interaction, users' multisensory perception of green space related characteristics are of great importance. The sensory perception of nature characteristics, especially, PSDs cannot be measured with landscape objective assessment approaches (De Jong et al., 2011). There are studies assessed the experienced green space characteristics, subjectively using selfreported measurement approach by lay-public participants (Lottrup et al., 2012; Stigsdotter and Grahn, 2011; Qiu and Nielsen, 2015; Memari et al., 2017). Studies supported the reliability of PSDs in measuring of green space characteristics using lay-public experiences, with similar outcomes, in different countries despite differences in culture and history (Memari et al., 2017; Sk ärb äck et al., 2015).

\section{3-Types of Restoration Experiences}

There are two groups of studies in evaluating the restorative effect of contact with nature as actual restoration experience studies and subjective restoration experience studies Hartig (2007). The actual restorative studies have attempted to find what happens between a person and an environment in the process of restoration experience (Hartig, 2007). The evidences of actual restoration outcomes come from laboratory or field experimental design studies. In assessment of the restorative potential of nature, prior to environmental treatment, mostly, studies used one of these antecedent mental fatigue conditions (controlled condition, fatigue induction, naturalistic induction, scenario approach and given level approach) to diminish respondents' ability for direct attention or to induce acute stressful experiences. Then, at different times of experiment (before, during or after exposure to nature), they measured respondents' state in the given environment by measures of direct attention, emotional state or through objective measurements of physiological activity systems.

There are a number of studies proposed validated tools that are more related to the concept of actual restoration experience in contact with nature. For example, Shortversion Revised Perceived Restorativeness Scale (SRPRS) proposed by Hartig et al. (1997b) to measure the recovery of directed attention from mental fatigue. Han (2003) suggested the Restorative Scale (RS) and Short-version Revised Restoration Scale (SRRS), in achievement of positive changes on ratings of emotional, physiological, cognitive and behavioral dimensions. In examining the restoration of different urban green spaces, Van den Berg et al. (2014) proposed the Restorative State Scale (RSS) with 9 items representing of the subject's overall experience. In measuring the 
possibility of likelihood of restoration, there are studies that suggested asking a single question of restoration experienced (e.g. "I would be able to rest and recover my ability to focus in this environment") (Nordh et al., 2009; Lindal and Hartig, 2013).

The subjective restoration studies, through the cross-sectional or longitudinal design approaches concern with the effect of repeated restorative experiences in people everyday contexts (Korpela et al., 2014; White et al., 2013). The focus in these studies is not on actual restoration in contact with nature. The term of stress in these studies referred to a long time stress experience not the acute stress, which is manipulated prior to nature intervention (Korpela et al., 2008). Research based on this approach measured outcomes of nature contact through several self-reported measurement instruments. For instance, White et al. (2013) measured the recalled feelings of restoration by rating on factors of calmness, relaxation, refreshment and revitalization. Restoration Outcome Scale (ROS) is another scale, which has been based by Hartig et al. (1998); Staats et al. (2003) and has been finalized by Korpela et al. (2008) in measuring of participants restoration experienced after visiting their favorite places. Using ROS, studies showed that restoration could be manifested through experiencing of 'relaxation and calmness', 'attention restoration' and 'clearing thoughts' (Korpela et al., 2008; Tyrväinen et al., 2014). There are two versions of ROS-6 items and ROS-9 items in use. Through ROS-9 items can measure higher levels of restoration experience, which is on personal development as subjective vitality and selfconfidence. These effects can occur by longer time involvement in restorative environments.

\section{4-Interaction with Nature}

In human-nature related studies, the relationship between nature and positive outcomes have mostly been investigated through passive and active approaches. Examples of passive interaction with nature can be through experiencing of the real or simulated image based models (e.g. videos, photos and paintings) or encountering with plants in indoor settings. Environmental health related studies have approved the reduction of subjects' stress and mental fatigue after representation of the simulated natural environments' images (e.g. Van den Berg et al., 2014; Felsten, 2009).

There are studies that highlighted the importance of having view out of window to restorative environments. Two famous addressed examples on the restorative outcomes of windows with view of natural elements have been provided by Ulrich (1984) in hospital and Kaplan (2001b) in living environments. In the study by Ulrich (1984), it showed that the patients who were in rooms with the nature view window had shorter post-operative care and took fewer pain-relieving medications than the patients in the rooms with brick blocks. In the study by Kaplan (2001b), views of natural elements from apartments' windows positively contributed to residents' satisfaction with their living area and their greater sense of well-being.

Following this line of research, studies on workplace settings found that viewing natural physical features including trees, lawns, flowering plants, water from windows have positively been associated with employees' perceived level of stress, well-being, high work ability and job satisfaction (e.g. Lottrup et al., 2015). In addition, in a high school setting, Matsuoka (2010) showed that exposure to the outdoor nature from indoor cafeteria as well as classroom windows was found to have positive influence on students' performance and fewer occurrences of criminal behavior. 
Being physically present in outdoor real-world settings and actively experiencing of natural features is another approach in exploring the human-nature relationship. The active exploring in the environment reflects the Gibson (1979)'s ecological approach to perception. In this perspective, landscape perception is the consequence of perception-action process (Heft, 2010) and involves the collaboration of movements of entire body and stimulation of the sensory system (Heft, 2010). The individuals' action in the environment facilitates detection of the environmental properties and receiving much valuable information that may provide opportunities for greater restoration experience. Literature review on field experimental studies has revealed many positive outcomes deriving from the direct way of being in environments with natural features (e.g. Beil and Hanes, 2013). An increasing amount of longitudinal and cross-sectional studies reported the importance of being exposed with natural elements (greenness/blueness) in individuals' everyday environments such as residential areas and workplaces. These studies addressed the cumulative restorative effects of repeated contact with nature on indicators of satisfaction, revitalization, tranquility, less risk of depression and anxiety (Gascon et al., 2015; Van den Berg et al., 2015).

Research on comparing both direct and indirect interactions with nature such as having a view of nature versus walking in that environment has demonstrated that they are not equivalent. For instance, in a study investigating the effects of outdoor walk in nearby nature and indoor walks through simulated scenes, Nisbet and Zelenski (2011) showed that individuals who participated in outdoor walks reported more positive outcomes than indoor walks did. In addition, Kjellgren and Buhrkall (2010) compared restoration outcomes of a natural environment with an indoor simulated of the same environment and found that, although the simulated natural environment facilitated stress reduction, the effect was significantly higher in contact with real natural environment. The same study showed that simulated natural environments' pictures have failed to stimulate sensory perceptions, while the natural environments significantly provided higher states of consciousness and energy. Although variety number of studies has documented the restorative potential of passive interaction with nature, the major problem with this way of interaction is that some important properties of restorative environments, which may have effect on experience of restoration, could be overlooked. Therefore, in assessment of restoration experience, direct interaction with nature rather than indirect interaction is more valuable in understanding the importance of open spaces qualities.

\section{5-Conclusion}

The psychological, physiological and emotional effect of natural environments has mostly been described based on the nature related theories of Biophilia hypothesis, SRT, ART and SET. Based on these theories, restoration experience in nature is a result of human connectedness to nature, stress alleviation, cognitive restoration through perceived restorativeness characteristics and a combination of certain green space characteristics. In this paper, different aspects of restoration experience through direct and indirect interaction with natural components were discussed. Moreover, the assessment methods in examining of different restorative outcomes were presented. Since, restoration experience refers to different concepts of stress alleviation and 
mental fatigue reduction, this paper provided a detail discussion on what kind of measurement method could be used for studies in these areas.

\section{References}

A. Abraham, K. Sommerhalder, and T. Abel. Landscape and Well-being: A Scoping Study on the Health-promoting Impact of Outdoor Environments. International Journal of Public Health, 55(1):59-69, 2010.

J. Francis, L. J. Wood, M. Knuiman, and B. \& Giles-Corti. Quality or Quantity? Exploring the Relationship between Public Open Space Attributes and Mental Health in Perth, Western Australia. Social Science \& Medicine, 74(10):15701577, 2012.

S. De Vries, S. M. Van Dillen, P. P. Groenewegen, and P. \& Spreeuwenberg. Streetscape Greenery and Health: Stress, Social Cohesion and Physical Activity as Mediators. Social Science \& Medicine, 94:26-33, 2013.

S. 'A. K. Johnsen. Exploring the Use of Nature for Emotion Regulation: Associations with Personality, Perceived Stress, and Restorative Outcomes. Nordic Psychology, 65(4):306-321, 2013.

R. S. Ulrich, R. F. Simons, B. D. Losito, E. Fiorito, M. A. Miles, and M. \& Zelson. Stress Recovery During Exposure to Natural and Urban Environments. Journal of Environmental Psychology, 11(3):201-230, 1991.

R. Kaplan and S. Kaplan. The Experience of Nature: A Psychological Perspective. CUP Archive, 1989.

T Hartig. Three Steps to Understanding Restorative Environments as Health Resources. In Open Space: People Space, pages 163-179. Taylor \& Francis, 2007.

T. Hartig and H. Staats. Linking Preference for Environments with their Restorative Quality. In Form Landscape Research to Landscape Planning: Aspects of Integration, Education and Application, pages 279-292. 2005.

J. Honold, T. Lakes, R. Beyer, and E. \& van der Meer. Restoration in Urban Spaces: Nature Views From Home, Greenways, and Public Parks. Environment and Behavior, 48(6): 1-30, 2015.

K. M. Korpela, M. Yl'en, L. Tyrväainen, and H. Silvennoinen. Determinants of Restorative Experiences in Everyday Favorite Places. Health \& Place, 14(4): 636652, 2008.

E. O. Wilson. Biophilia. Cambridge, Mass.: Harvard University Press., 1984.

E. K. Nisbet, J. M. Zelenski, and S. A. Murphy. Happiness is in our Nature: Exploring Nature Relatedness as a Contributor to Subjective Well-Being. Journal of Happiness Studies, 12(2):303-322, 2011.

D. Haluza, S. Simic, J. H”oltge, R. Cervinka, and H. Moshammer. Connectedness to Nature and Public (Skin) Health Perspectives: Results of a Representative, Population-Based Survey among Austrian Residents. International Journal of Environmental Research and Public Health, 11(1):1176-1191, 2014.

C. Capaldi, R. L Dopko, and J. M Zelenski. The Relationship between Nature Connectedness and Happiness: A Meta-analysis. Frontiers in Psychology, 5 (September): 1-15, 2014.

F. S Mayer and C. M Frantz. The Connectedness to Nature Scale: A Measure of Individuals' Feeling in Community with Nature. Journal of Environmental Psychology, 24(4):503-515, 2004. 
J. L. Perrin and V. A. Benassi. The Connectedness to Nature Scale: A Measure of Emotional Connection to Nature? Journal of Environmental Psychology, 29(4):434-440, 2009.

K. T. Han. A Reliable and Valid Self-rating Measure of the Restorative Quality of Natural Environments. Landscape and Urban Planning, 64(4):209-232, 2003.

R S Ulrich. View through a Window may Influence Recovery from Surgery. Science, 224(1984):420-421, 1984.

G. N. Bratman, G. C. Daily, B. J. Levy, and J. J. \& Gross. The Benefits of Nature Experience: Improved Affect and Cognition. Landscape and Urban Planning, 138:41-50, 2015.

L. Tyrväinen, A. Ojala, K. Korpela, T. Lanki, Y. Tsunetsugu, and T. Kagawa. The Influence of Urban Green Environments on Stress Relief Measures: A Field Experiment. Journal of Environmental Psychology, 38:1-9, 2014.

B. J. Park, K. Furuya, T. Kasetani, N. Takayama, T. Kagawa, and Y. Miyazaki. Relationship between Psychological Responses and Physical Environments in Forest Settings. Landscape and Urban Planning, 102(1):24-32, 2011.

M. R. Marselle, K. N. Irvine, and S. L. Warber. Examining Group Walks in Nature and Multiple Aspects of Well-being: A Large-scale Study. Ecopsychology, 6(3):134-147, 2014.

R. S. Ulrich. Visual Landscapes and Psychological Wellbeing. Landscape Research, 4(1):17-23, 1979.

R. Kaplan. The Role of Nature in the Context of the Workplace. Landscape and Urban Planning, 26(1):193-201, 1993.

S. Kaplan. The Restorative Benefits of Nature: Toward an Integrative Framework. Journal of Environmental Psychology, 15(3):169-182, 1995.

S. Kaplan, L. V. Bardwell, and D. B. Slakter. The Museum as a Restorative Environment. Environment and Behavior, 25(6):725-742, 1993.

T. Hartig, F. G. Kaiser, and P. A Bowler. Further Development of a Measure of Perceived Environmental Restorativeness. Institutet f"or bostadsforskning, 1997a.

M. G. Berman, J. Jonides, and S. Kaplan. The Cognitive Benefits of Interacting with Nature. Psychological Science, 19(12):1207-1212, 2008.

M. Pilotti, E. Klein, D. Golem, E. Piepenbrink, and K. Kaplan. Is Viewing a Nature Video After Work Restorative? Effects on Blood Pressure, Task Performance, and Long-Term Memory. Environment and Behavior, 47(9): 1-23, 2014.

R. K. Raanaas, K. H. Evensen, D. Rich, G. Sjøstrøm, and G. Patil. Benefits of Indoor Plants on Attention Capacity in an Office Setting. Journal of Environmental Psychology, 31(1):99-105, 2011.

S. Kaplan. Meditation, Restoration, and the Management of Mental Fatigue. Environment and Behavior, 33(4):480-506, $2001 \mathrm{a}$.

K. Laumann, T. Gärling, and K. Stormark. Rating Scale Measures of Restorative Components of Environments. Journal of Environmental Psychology, 21 (1):3144, 2001.

K. L. Bagot, F. C. L. Allen, and S. Toukhasati. Perceived Restorativeness of Children's School Playground Environments: Nature, Playground Features and Play Period Experiences. Journal of Environmental Psychology, 41:1-9, 2015.

R. Kaplan. The Nature of the View from Home: Psychological Benefits. Environment and Behavior, 33(4):507-542, $2001 \mathrm{~b}$.

T. R. Herzog, P. Maguire, and M. B. Nebel. Assessing the Restorative Components of Environments. Journal of Environmental Psychology, 23(2):159-170, 2003. 
R. Pals, L. Steg, F.W. Siero, and K.I. van der Zee. Development of the PRCQ: A Measure of Perceived Restorative Characteristics of Zoo Attractions. Journal of Environmental Psychology, 29(4):441-449, 2009.

S. Kaplan and C. Peterson. Health and Environment: A Psychological Analysis. Landscape and Urban Planning, 26(1-4):17-23, 1993.

M. Scopelliti and M. Vittoria Giuliani. Choosing Restorative Environments Across the Lifespan: A Matter of Place Eexperience. Journal of Environmental Psychology, 24(4):423-437, 2004.

C. T. Ivarsson and C. M. \& Hagerhall. The Perceived Restorativeness of Gardens Assessing the Restorativeness of a Mixed Built and Natural Scene Type. Urban Forestry \& Urban Greening, 7(2):107-118, 2008.

M. Marselle, K. Irvine, A. Lorenzo-Arribas, and S. Warber. Moving beyond Green: Exploring the Relationship of Environment Type and Indicators of Perceived Environmental Quality on Emotional Well-Being following Group Walks. International Journal of Environmental Research and Public Health, 12(1):106130, 2015.

M. R. Marselle, K. N. Irvine, A Lorenzo-Arribas, and S. L. Warber. Does Perceived Restorativeness Mediate the Effects of Perceived Biodiversity and Perceived Naturalness on Emotional Well-Being Following Group Walks in Nature? Journal of Environmental Psychology, 46:217-232, 2016.

J. A. Hipp, G. B. Gulwadi, S. Alves, and S. Sequeira. The Relationship between Perceived Greenness and Perceived Restorativeness of University Campuses and Student-Reported Quality of Life. Environment and Behavior, 48(10): 1292-1308, 2016.

T. Hartig, K. Korpela, and G.W Evans. A Measure of Restorative Quality in Environments. Scandinavian Housing and Planning Research, 14(4):175-194, 1997b.

T. Hartig, M. Mang, and G. W. Evans. Restorative Effect of Natural Environment Experiences. Environment and Behavior, 23(1):3-26, 1991.

T. Hartig, G. W Evans, and T G̈arling. Validation of a Measure of Perceived Environmental. University of G oteborg, Department of Psychology, 1996.

K. L. Bagot. Perceived Restorative Components: A Scale for Children. Children, Youth and Environments, 14(1):107-129, 2004.

X. Y. Lehto. Assessing the Perceived Restorative Qualities of Vacation Destinations. Journal of Travel Research, 52(3):325-339, 2013.

M. Pasini, R. Berto, M. Brondino, R. Hall, and C. Ortner. How to Measure the Restorative Quality of Environments: The PRS-11. Procedia Social and Behavioral Sciences, 159:293-297, 2014.

A. E. Van den Berg, A. Jorgensen, and E. R. Wilson. Evaluating Restoration in Urban Green Spaces: Does Setting Type Make a Difference? Landscape and Urban Planning, 127:173-181, 2014.

P. Grahn, C. Tenngart, I. Ulrika, and I Bengtsson. Using Affordances as a Healthpromoting Tool in a Therapeutic Garden. In Innovative Approaches to Researching Landscape and Health, pages 116-154. 2010.

J. Stoltz, Y. Lundell, E. Skärbäck, M. A. van den Bosch, P. Grahn, E. M. Nordstr"om, and A. Dolling. Planning for Restorative Forests: Describing Stressreducing Qualities of Forest Stands Using Available Forest Stand Data. European Journal of Forest Research, 135(5):803-813, 2016. 
P Grahn and U. K. Stigsdotter. The Relation between Perceived Sensory Dimensions of Urban Green Space and Stress Restoration. Landscape and Urban Planning, 94(3):264-275, 2010.

P. Grahn, U. A. Stigsdotter, and A. M Berggren-Barring. A Planning Tool for Designing Sustainable and Healthy Cities. The Importance of Experienced Characteristics in Urban Green Open Spaces for People's Health and WellBeing. In Quality and Significance of Green Urban Areas, pages 29-38. Van Hall Larwnstein University of Professional Education, Netherlands, 2005.

P. Grahn. Landscapes in Our Minds: People's Choice of Recreative Places in Towns. Landscape Research, 16(1):11-19, 1991.

A. V. Herzele and T. Wiedemann. A Monitoring Tool for the Provision of Accessible and Attractive Urban Green Spaces. Landscape and Urban Planning, 63:109-126, 2003.

K. Maikov, S. Bell, and K. Sepp. An Evaluation of the Design of Room Characteristics of a Sample of Healing Gardens. WIT Transactions on Ecology and the Environment, I14:223-232, 2008.

M. Kytta and M. Kahila. The Perveived Quality Factors of the Environment and their Ecoefficient Acceccibility. Forests, Trees and Human Health and Wellbeing, 2005.

L. Tyrväinen, K. Mäkinen, and J. Schipperijn. Tools for Mapping Social Values of Urban Woodlands and Other Green Areas. Landscape and Urban Planning, 79(1):5-19, 2007.

J. Bjork, M. Albin, P. Grahn, H. Jacobsson, J. Ardo, J. Wadbro, P. O. Ostergren, and E. Skarback. Recreational Values of the Natural Environment in Relation to Neighbourhood Satisfaction, Physical Activity, Obesity and Wellbeing. Journal of Epidemiology \& Community Health, 62(4):e2, 2008.

P. Grahn and A. M. Berggren-B̈arring. Experiencing Parks. Man's Basic Underlying Concepts of Qualities and Activities and their Impact on Park Design. Ecological Aspects of Green Areas in Urban Environments, pages 97-101, 1995.

K. De Jong, M. Albin, E. Ska r̈bӓck, P. Grahn, J. Wadbro, J. Merlo, and J. Bj ork. Area-aggregated Assessments of Perceived Environmental Attributes May Overcome Single-source Bias in Studies of Green Environments and Health: Results from a Cross-sectional Survey in Southern Sweden. Environmental Health, 10(1):1-11, 2011.

L. J. Wolf, S. zu Ermgassen, A. Balmford, M. White, and N. Weinstein. Is Variety the Spice of Life? An Experimental Investigation into the Effects of Species Richness on Self-Reported Mental Well-Being. PloS one, 12(1): e0170225, 2017.

K. K. Peschardt and U. K. Stigsdotter. Associations between Park Characteristics and Perceived Restorativeness of Small Public Urban Green Spaces. Landscape and Urban Planning, 112(1):26-39, 2013.

U. K. Stigsdotter, S. S. Corazon, U. Sidenius, A. D. Refshauge, and P. Grahn. Forest Mental Health PromotionUsing Perceived Sensory Dimensions to Elicit Restorative Responses. Landscape and Urban Planning, 160:1-15, 2017.

S. Memari, M. Pazhouhanfar, and A. Nourtaghani. Relationship between Perceived Sensory Dimensions and Stress Restoration in Care Settings. Urban Forestry and Urban Greening, 26(May):104-113, 2017.

M. Annerstedt, P. O. O־stergren, J. Bj ork, P. Grahn, E. Sk ärbäack, and P. \& W"ahrborg. Green Qualities in the Neighbourhood and Mental Health Results from a Longitudinal Cohort Study in Southern Sweden. BMC Public Health, 12(1):337, 2012. 
M.A.VandenBosch,P.O.O stergren,P.Grahn,E.Sk ärb äk,andP.W"ahrborg. Moving to Serene Nature May Prevent Poor Mental Health Results from a Swedish Longitudinal Cohort Study. International Journal of Environmental Research and Public Health, 12(7):7974-7989, 2015.

K De Jong, M Albin, E Sk ärb äck, P Grahn, and J Bj ork. Perceived Green Qualities were Associated with Neighborhood Satisfaction, Physical Activity, and General Health: Results from a Cross-Sectional Study in Suburban and Rural Scania, Southern Sweden. Health and Place, 18:1374-1380, 2012.

S. M. Van Dillen, S. de Vries, P. P. Groenewegen, and P. Spreeuwenberg. Greenspace in Urban Neighbourhoods and Residents' Health: Adding Quality to Quantity. Journal of Epidemiology and Community Health, 66(6):e8-e8, 2012.

L. Qiu and A. B Nielsen. Are Perceived Sensory Dimensions a Reliable Tool for Urban Green Space Assessment and Planning? Landscape Research, 40(7): 834 $854,2015$.

M. Hofmann, J. R. Westermann, I. Kowarik, and E. \& van der Meer. Perceptions of Parks and Urban Derelict Land by Landscape Planners and Residents. Urban Forestry and Urban Greening, 11(3):303-312, 2012.

D. Karmanov and R. Hamel. Assessing the Restorative Potential of Contemporary Urban Environment (s): Beyond the Nature Versus Urban Dichotomy. Landscape and Urban Planning, 86(2):115-125, 2008.

L. Lottrup, U. K. Stigsdotter, H. Meilby, and S. S Corazon. Associations between Use, Activities and Characteristics of the Outdoor Environment at Workplaces. Urban Forestry \& Urban Greening, 11(2):159-168, 2012.

U. K Stigsdotter and P. Grahn. Stressed Individuals' Preferences for Activities and Environmental Characteristics in Green Spaces. Urban Forestry \& Urban Greening, 10(4):295-304, 2011.

Erik Sk äb äck, Lu Wen, Silviia Aleksandrova, and Patrik Grahn. The Serene and Other Affordances in Demanding Contexts. IFLA 52nd World Congress, pages 1 9, 2015.

H. Nordh, T. Hartig, C.M. Hagerhall, and G. Fry. Components of Small Urban Parks that Predict the Possibility for Restoration. Urban Forestry \& Urban Greening, 8(4):225-235, 2009.

P. J. Lindal and T. Hartig. Architectural Variation, Building Height, and the Restorative Quality of Urban Residential Streetscapes. Journal of Environmental Psychology, 33:26-36, 2013.

K. Korpela, K. Borodulin, M. Neuvonen, O. Paronen, and L. Tyrv äinen. Analyzing the Mediators between Nature-based Outdoor Recreation and Emotional Wellbeing. Journal of Environmental Psychology, 37:1-7, 2014.

M. P. White, S. Pahl, K. Ashbullby, S. Herbert, and M. H. Depledge. Feelings of Restoration from Recent Nature Visits. Journal of Environmental Psychology, 35:40-51, 2013.

T. Hartig, K. Lindblom, and K. Ovefelt. The Home and Nearhome Area Offer Restoration Opportunities Differentiated by Gender. Scandinavian Housing and Planning Research, 15(4):283-296, 1998.

H. Staats, A. Kieviet, and T. Hartig. Where to Recover from Attentional Fatigue: An Expectancy-value Analysis of Environmental Preference. Journal of Environmental Psychology, 23(2):147-157, 2003.

G Felsten. Where to Take a Study Break on the College Campus: An Attention Restoration Theory Perspective. Journal of Environmental Psychology, 29 (1):160-167, 2009. 
L Lottrup, U. K. Stigsdotter, H Meilby, and A. G Claudi. The Workplace Window View: A Determinant of Office Workers' Work Ability and Job Satisfaction. Landscape Research, 40(1):57-75, 2015.

R. H. Matsuoka. Student Performance and High School Landscapes: Examining the Links. Landscape and Urban Planning, 97(4):273-282, 2010.

J. J. Gibson. The Ecological Approach to Visual Perception. Boston: Houghton Mifflin, 1979.

H. Heft. Affordances and the Perception of Landscape: An Inquiry into Environmental Perception and Aesthetics. In Innovative Approaches to Researching Landscape and Health., pages 9-32. 2010.

K. Beil and D. Hanes. The Influence of Urban Natural and Built Environments on Physiological and Psychological Measures of Stress A Pilot Study. International Journal of Environmental Research and Public Health, 10(4): 1250-1267, 2013.

M. Gascon, M. Triguero-mas, D. Mart'inez, P. Dadvand, J. Forns, A. Plas`encia, and M. J. Nieuwenhuijsen. Mental Health Benefits of Long-Term Exposure to Residential Green and Blue Spaces : A Systematic Review. International Journal of Environmental Research and Public Health, 12(4):4354-4379, 2015.

M. Van den Berg, W. Wendel-Vos, M. van Poppel, H. Kemper, W. van Mechelen, and J. \& Maas. Health Benefits of Green Spaces in the Living Environment: A Systematic Review of Epidemiological Studies. Urban Forestry \& Urban Greening, 4(14):806-816, 2015.

E. K. Nisbet and J. M. Zelenski. Underestimating Nearby Nature: Affective Forecasting Errors Obscure the Happy Path to Sustainability. Psychological Science, 22(9):1101-1106, 2011.

A. Kjellgren and H. Buhrkall. A Comparison of the Restorative Effect of a Natural Environment with that of a Simulated Natural Environment. Journal of Environmental Psychology, 30(4):464-472, 2010. 